\title{
Gait rehabilitation with a robotic dog
}

\author{
M. Naganuma $^{1 *}$, E. Ohkubo ${ }^{2}$, R. Kimura ${ }^{1}$, M. Watanabe ${ }^{3}$ and N. Kato ${ }^{3}$ \\ ${ }^{1}$ Teikyo University of Science, Adachi, Tokyo, Japan \\ ${ }^{2}$ Teikyo Junior College, Shibuya, Tokyo, Japan \\ ${ }^{3}$ Tokorozawa Loyal Hospital, Tokorozawa, Saitama, Japan \\ *Corresponding author (naganuma@ntu.ac.jp)
}

\begin{abstract}
Purpose Gait rehabilitation is a fundamental requirement to avoid disuse syndrome for the long-term hospitalized person such as cerebral infarction patient or patient with dementia. In general gait rehabilitation is monotonous and dull, so some entertainment or a sense of achievement is helpful to maintain motivation during the daily exercise. This paper proposes an effective gait-rehabilitation system using a robotic dog supporting individual initiatives of the patient. Method Walking with a living dog is one way to achieve this; however as an intervention it is limited to mildly symptomatic patients because a real dog risks sudden lunging. We used a robotic dog instead of a living dog for relatively critical patients. We have studied robot-assisted activity and therapy using robotic animals in the elderly nursing home in order to improve residents' quality of life (QOL). In the case of a man-made robot, aforementioned risks can be ignored. Moreover, it is an advantage that an in-house walking exercise with the dog is possible. The basic concept of this proposal is co-creation of steps between the elderly and dog. That is, the real-time stabilometric signal of the elderly was measured and analyzed to extract a single step and forwarded to the robotic dog to make a step. Since commercially available amusement products pass through the safety review of public administration, the proposed system was composed of those products as a hardware tool. More specifically, entertainment robot AIBO of Sony ERC was adopted as the robotic dog and Nintendo's Wii-balance-board is used as the stabilometric human interface devices. All signals are transmitted by conventional Bluetooth and LAN systems. Results \& Discussion Figure 2 shows the block diagram and enforcement scenes of gaiting exercise at standing and sitting positions, respectively. The patients both suffered from dementia and made their own steps in a positive manner shown by arrows in the figure. Such positive attitude was uncommon in the conventional daily exercise. Functions for walking back and forth walk, and turning right and left are installed. It is noteworthy that the standing patient tempts the dog to move toward another patient and the patient calls the robot to her. This means there a co-creation field is created through the robotic dog. This was followed by a chat between the patients. In the case of the sitting patient, although for her it is very hard exercise to raise her foot from the floor, she made several steps on her own initiative successively after the physical therapist guided her how to walk by patting and lifting her right and left knees alternately. The position of the center of gravity and command signal were logged every $0.1 \mathrm{sec}$ in the control PC and available to be analyzed afterward. For example, the change of exhaustion during exercise was estimated from the magnitude and regularity of steps. Since all hardware tools can be packed in a suitcase the proposed gait exercise can be easily done everywhere.
\end{abstract}

Keywords: robotics, rehabilitation, nursing, dementia, gait, co-creation, therapy

\section{INTRODUCTION}

Interaction between a robot and an aged person produces effects in three domains, physical, mental, and social. In relation to the physical domain, industrial robot technologies are being applied to provide practical equipment that assists walking, bathing, and other daily physical activities, but research on the application of robotics in relation to the metal and social domains has been more limited. Recently, however, robot-assisted activity/therapy has been receiving considerable attention in all three domains $^{1-4}$. While animal-assisted activity/therapy has a long history and much research has been accumulated on its utility and effectiveness, it has some limitations. We have been conducting studies on robot-assisted activity from the viewpoint of overcoming the limitations by replacing the animal with a robot $^{5}$. These limitations arise from the fact that the animal is a living creature and therefore poses problems of infection, feeding and excretion, and needing a handler. In addition, we must be mindful of animal abuse. All of these problems can be solved when the animal is replaced with a robot. One of the issues being focused on in robotics research is how to make the robot show elements of a living creature.

In this paper, we discuss how the use of an animal robot can induce aged persons to complete monotonous walking exercise. The important aspect of the proposed approach to walking rehabilitation is that the aged persons control the robots independently.

MERITS OF USERS CONTROLLING AN ANIMAL ROBOT
FOR WALKING REHABILITATION Aged persons staying in nursing homes or hospitals over the long term are at risk of serious accidents 
such as falls due to decline in physical functioning. To help prevent this, residential facilities offer a variety of physical exercises to augment the medical treatments the residents receive. Simple standing and walking are among the most basic and effective of these exercises. A number of positive effects can be expected if residents are able to walk around the home or hospital: it expands their active range, which in turn improves their sociality, an important factor for aged residents who can readily spend time on their one. Walking exercise is therefore of crucial importance. However, such exercise becomes a chore if there is little sense of purpose for it.

Most aged persons have some experience of walking a living dog. Even for those without physically impairment, walking a dog brings health benefits such as prevention of metabolic syndrome. It can be difficult and even dangerous, however, for patients who are largely housebound due to functional deterioration to try to walk outdoors with a living dog. In particular, patients with loss of motor function following cerebral infarction have to begin with very basic exercise like taking one step at a time when walking. Such walking exercise is monotonous and hard work, and residential facilities are concerned about how to motivate residents $w$ to continue exercising. If walking exercise could be made entertaining, the residents' attitude toward exercise could be changed from a passive to active one. As an example, Figure 1 shows the robot walking game called the "AIBO Derby" that uses Sony Corporation's robotic dog AIBO. The robot is equipped with a program to chase a pink-colored object. Four AIBOs are lined up at the start line, as in a horse race, and they "run" on a course prepared on a table-tennis table toward their 'owners'. The users hold and show a pinkcolored ball to their own AIBO so that their AIBO walks faster toward them. Competing in the race leads to positive feelings among the participants and most of them are unconsciously doing physical exercise through standing, holding the ball, and waving the ball as a stimulus. In this exercise, in addition to producing the desired physical effects, the residents' sociality will also be improved through mutual communication during the game.

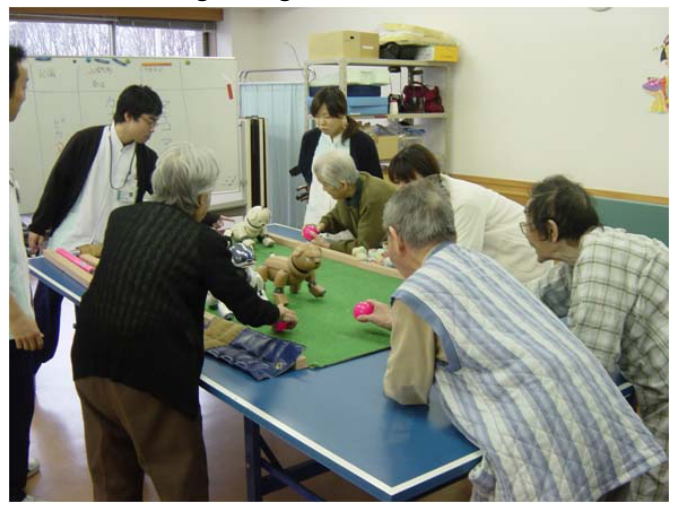

Fig. 1 The "AIBO Derby" game using AIBO robotic dogs

\section{CONFIGURATION OF THE PROPOSED SYSTEM Outline of the system}

The proposed system is composed of three hardware components: the human interface device (HID), the control server computer, and the robot. All of the hardware devices can be transported in a mediumsized suitcase. The HID senses the resident's physical action and sends data to the server. The server computer analyzes the data and converts it to a control command which is then sent to the robot. The control command activates the robot's actions that are programmed and installed in the body of the robot in advance. A block diagram of the system is shown in Fig. 2. In order to keep spatial freedom

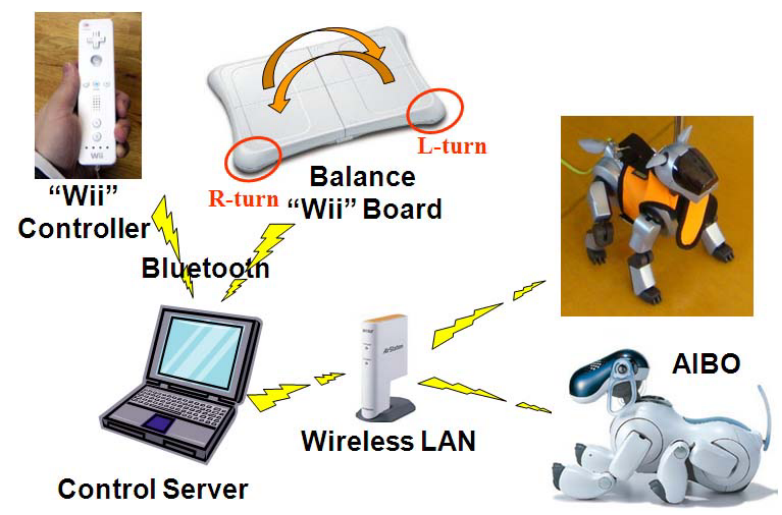

Fig. 2 Block diagram of the proposed system

and safety, all electric data signals are transmitted through wireless links and all of the devices are driven by a battery to avoid accidents involving electric cables. We used commercially available hardware devices when creating the entire system for the following reasons. First, the devices already on the market have passed a public safety review so they can be used in public spaces such as hospitals and nursing homes for the elderly. Also, commercially available amusement tools are ergonomically sophisticated and are a comfortable fit for aged persons. Second, just the distribution of the software allows therapists to quickly and easily try the robotassisted therapy in their work setting at a moderate price. This is important because as awareness of robot-assisted activity/therapy is much lower than that of animal-assisted activity/therapy, we need to encourage therapists to try it and see its effectiveness for themselves.

\section{Human interface device}

We adopted the operation terminal of a TV game supplied by Nintendo as the HID. The HID needed to have functions that aged persons could operate easily to control the robot. Two types of HID were selected for use. The first type is the hand-held acceleration sensing HID called the "Wii remote controller", shown in the top left of Fig. 2. This HID was selected for users who can walk unassisted. 
The user can send a signal to make the robotic dog walk simply by swinging the device and then follows the robot on foot when it starts to walk ${ }^{6}$. The HID, which can be fixed to a waist belt, can also move the robot forward by acceleration of the moving trunk when tuned to a higher sensitivity.

The second type of HID used was the "Wii Balance Board", shown in the upper center of Fig. 2. This HID was selected for users who have difficulty standing and walking by themselves. This HID is a kind of stabilometer and is equipped with four load sensors, one at each corner of the board. The user makes the robotic dog walk by simply making steps on the board. The relationship between the user's single step and robot's single step can be tuned by the control program.

\section{Control server}

The HID device and the control server are connected by a Bluetooth wireless system $(2.4 \mathrm{GHz}, 1 \mathrm{Mbps})$. The specs for a conventional PC are sufficient for the control server. A netbook type PC with touch panel operation, in this case the Fujitsu FMV-LOOX series, was used.

When using the first type of HID, the handheld HID, the control server analyzes the acceleration strength detected by the HID and sends a walk command to the robot when it exceeds the preliminarily given threshold. The robotic dog then walks a certain number of steps. The threshold value and the number of steps can be set up through the server console.

When using the second type of HID, the balance board HID, every 100 ms the server program calculates the coordinate value of the position of the center of gravity based on the data from the four load sensors in the board. It determines whether the user is sending a signal for the robot to make a step or not from the user's change in the position of the center of gravity. In detail, just after the board is switched on and before loading, the four sensors are calibrated so that the center of gravity lies at the center of the board. A step is considered made when the center of gravity passes over a virtual vertical threshold line that lies at the right or left center of the board, under the condition that the center of gravity remains for a certain period in the right or left region of the board before crossing over. This condition is in place to avoid a crossing error caused by noise signals. In addition, there are special areas configured to send signals to turn the robot right and left: a right (left) command is generated if the center of gravity stays in the right (left) front area of the board.

For users who cannot maintain a standing position with the balance board HID, a mode with much higher sensitivity is available, called the "on-chair" mode. Both the standing and on-chair modes have heavy, medium, and light exercise settings, enabling the user to select from six difficulty levels of exercise. The position of the center of gravity is displayed on the console screen in-situ as a real-time position or as a locus. These selections are all made on the server console, named the "AlBOard" (Fig. 3). All temporal data including the center of gravity and induced commands are automatically saved every $100 \mathrm{~ms}$ into a log file that can be analyzed later.

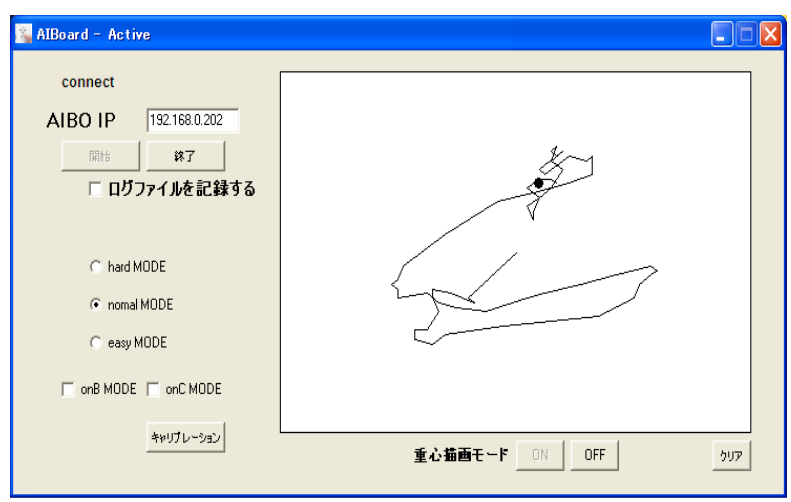

Fig. 3 Control console, the "AIBOard"

\section{Robotic dog}

The AIBO ERS-200 series and ERS-7 series supplied by Sony Corporation were used as the robotic dog. AIBO ERS210 and ERS-7 are shown in the top and bottom right of Fig. 2, respectively. These robots were chosen from those available on the market because they are equipped with wireless LAN hardware and an IP address can be assigned, and the robot's basic behavior can be programmed by the user using the supplied programming platform. This enables the user to control the robot's motion, such as back and forth steps and right and left turns, based on the commands transmitted from the server. A dog lead can be connected to the robotic dog to help promote the walking exercise if preferred.

\section{Operation procedure}

The devices are switched on and the wireless connection is confirmed on the server console. The IP address of the AIBO is then input so that the data communication channel from the HID to the AIBO is kept logically active. The time needed for this procedure is $5 \mathrm{~min}$ at most. When using the balance board as the HID, the AIBO waits in the sitting position with no load on the board, then stands up and waits when the user places weight on the board.

\section{RESULTS}

\section{Operation with the handheld HID}

The proposed system was used in a nursing home for the elderly, as shown in Fig. 4. It was observed that an ambulant user followed the robotic dog that he controlled through simple swinging of the HID. The user who had mild dementia also talked with 
nursing staff about a dog that he used to feed in the past. In this instance, the original purpose of the proposed system was achieved as the robotic dog prompted the user to walk voluntarily around the home. It became clear, however, that since the walking rate of the AIBO is rather slow, when using the handheld HID effectiveness would be limited to users who have major difficulty in walking unassisted.

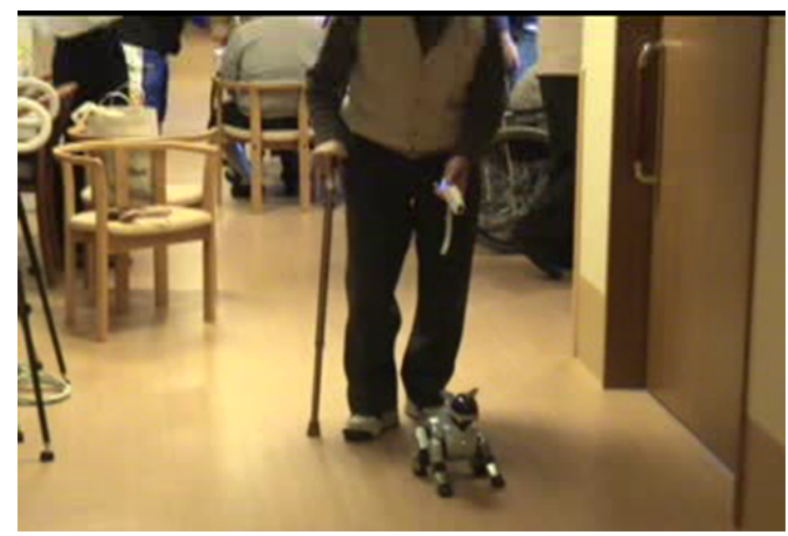

Fig. 4 Walking exercise using the handy HID

\section{Operation with the balance board HID}

Walking exercise using the balance board HID is suitable for users who cannot walk without an assistive device. The user makes steps on the board placed within a walking frame (Fig. 5). The recorded loci of the center of gravity for a younger, healthy male user (90 kg body weight) and for an aged user (45 kg body weight) are shown in Fig. 6. The loci correspond to position recorded every $100 \mathrm{~ms}$ and lines connect the positions. For the case of the younger user, clear weight shifts can be seen to the right upper and left upper quadrants to turn the robot and normal shifts between the right and left sides of the board enabled the AIBO to walk straight ahead. For the aged user, however, the weight shift was insufficient to complete turns of the robot and making the robot walk continuously in a straight line by weight shifting from right to left was clearly very difficult.

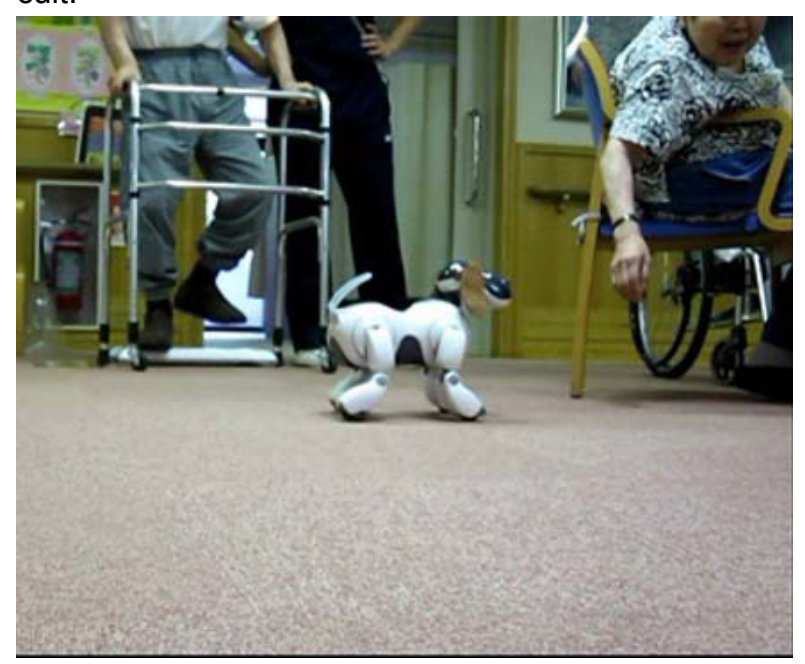

Fig. 5 Walking exercise using the board HID

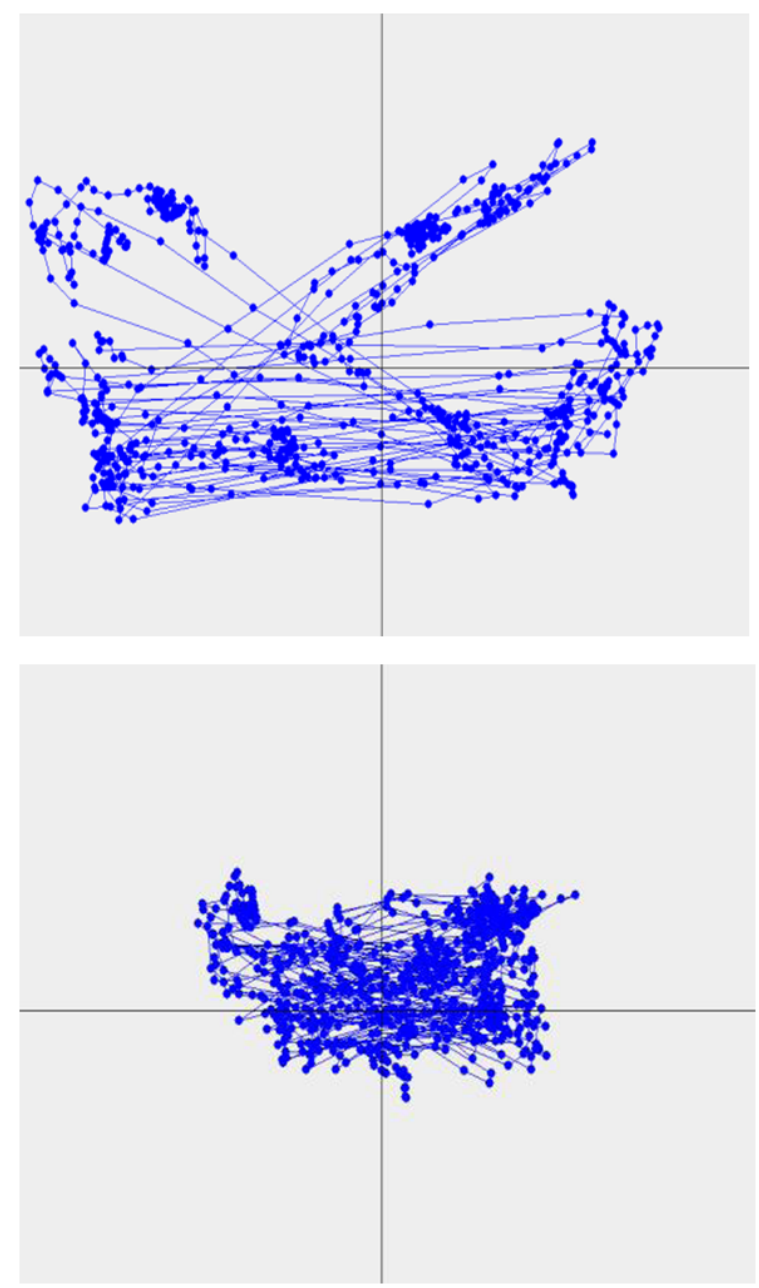

Fig. 6 Loci of the center of gravity

Top: younger, healthy male user, Bottom: aged user

One of the problems associated with using stepping on the balance board HID to operate the system is that the user cannot move forward in the same way as in normal walking. Figure 7 compares theloci of two aged users, $A$ and $B$, with similar body weight. The range of B's forward and backward weight shifts is obviously larger than A's while the right-to-left weight shifts are similar for both users. Figure 8 shows weight shifts occurring over a 1-min period (taken from Fig.7) for users A (blue line) and B (red line). User B moves slightly forward over time and returns to the original position several times whereas user A maintains relatively stable stepping.

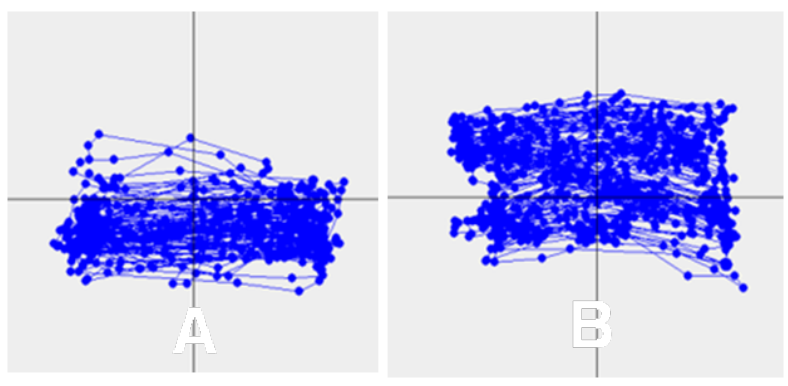

Fig.7 Loci of the center of gravity for aged users $A$ and $B$ 


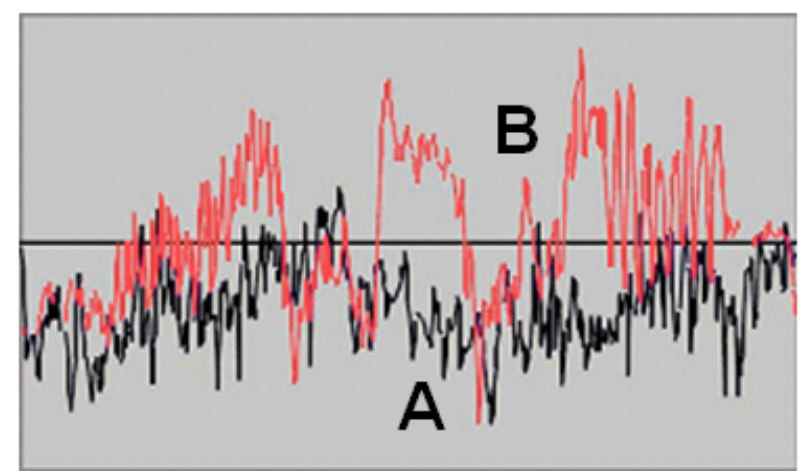

Fig. 8 Temporal change in the position of the center of gravity for aged users $A$ (black) and $B$ (red) over a 1min period

The typical right-to-left weight shift over a 1-s period is shown in Fig. 9. The moment a step occurs is

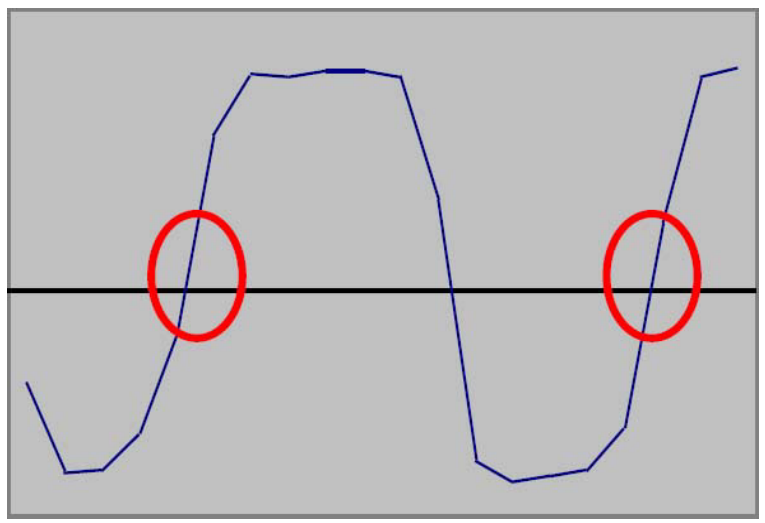

Fig. 9 Typical pattern of movement of the center of gravity, where a circle denotes the time a step is made when the center of gravity passes over the center threshold line

defined as the time when the center of gravity passes over the center threshold line (red circle). Figure 10 shows the change in stepping rate over a 4-min period. Stepping begins with a slow repetition rate of $1.4 \mathrm{~s}$, then becomes stable from about $1 \mathrm{~s}$ and becomes disordered around midway through the period.

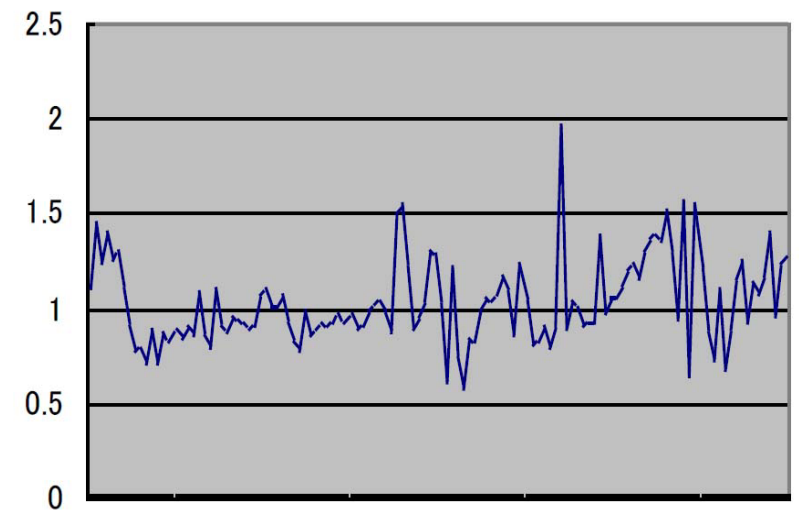

Fig. 10 Temporal change in stepping interval (sec.) over a 4-min period

Simultaneously recorded video images show that the user initially took rhythmical and stable steps elevat- ing the foot, but this elevation gradually reduced and weight shift was then made by moving the trunk toward the right and left. he book will be printed in black and white.

\section{Discussion}

Most participants in this preliminary study to examine the possibilities of the proposed system had dementia with a score on the Hasegawa Dementia ScaleRevised (HDS-R) ranging from 5 to 25 (30 = healthy, $0=$ severe dementia). The system, which enables users to control the robot by swinging the hand or making steps, appeared to be useful with these participants. The proposed walking exercise would also be useful for rehabilitation of spatial configuration recognition. On the balance board, the users remain in a fixed location while the robot moves and points in various directions. To turn the robot in the chosen direction, the user has to construct the virtual spatial configuration that includes both the robotic dog and himself. When both parties are pointing in the same direction, it is relatively easy to make the robot turn because the direction of the robot and user coincide. This is identical to the case using the handheld HID when the user swings it and follows the robot. When the user is facing the robot it becomes more difficult to make the robot turn because right for the robot corresponds to left for the user. The user must recognize this and make the necessary movement in order for the robot to turn correctly.

The demand for the proposed walking exercise system was strong among the participants who could not maintain a standing position. The trial for one of these participants is shown in Fig. 11. The user sits on the chair and makes a step on the board. The weight of just the leg itself can be used in this instance to operate the robot, as opposed to the whole

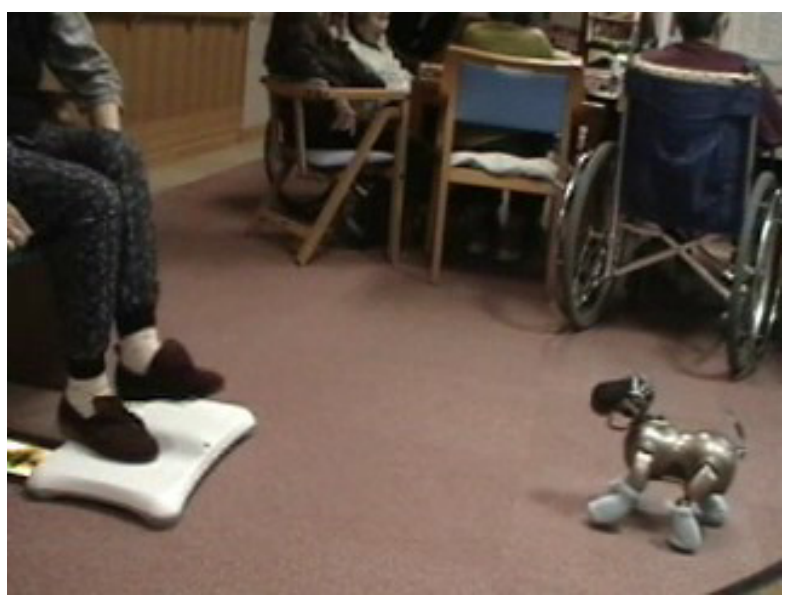

Fig. 11 Operation mode while seated

body weight being used by users in the standing position. For seated users it is necessary therefore to increase the sensitivity of the load sensors and to reduce the noise to avoid malfunction. As shown in 
Fig. 11, the robot could be operated from the chair by stepping after tuning of the stepping decision algorithm.

It was seen in this study that although most patients did not view the robotic dog as a living creature, their voluntary behavior was still induced it. It seems that they viewed the robot as machine-like but not a real machine, which would be due to the phenomenon of embodiment that comes from the robots dog-like appearance and its animal-like behavior. As evidence to support this, users often addressed the robot directly and voluntarily. Although animalassisted activity using living animals shows promise for improving the quality of life of aged persons, it still requires a handler to be present during rehabilitation exercises for aged persons with physical and cognitive impairment. Robots are able to compensate for this limitation and also can likely maintain quality of life when used complementarily with animals.

\section{CONCLUSION}

Walking rehabilitation exercise using the robotic dog AIBO was proposed. The entire system including the HID was prepared from commercially available products. The preliminary trial showed possibilities for its use with aged persons with quite severe physical and cognitive decline and shows promise to assist in their rehabilitation.

\section{ACKNOWLEDGEMENT}

This work was supported by the Grants-in-Aid for Scientific Research of JSPS (\#23500662)

\section{References}

1. Shibata, T., "An overview of Human Interactive Robots for Psychological Enrichment", Proc. the IEEE, Vol.92, pp.1749-1758, 2004.

2. Tamura, T., Yonemitsu, S., Itoh, A., Oikawa, D., Kawakami, A., Higashi, Y., Fujimooto, T., Nakajima, K., "Is an Entertainment Robot Useful in the Care of Elderly People With Severe Dementia?", J. Gerontology Series A: Biological Sciences and Medical, Vol.59, pp. 83-85, 2004.

3. Ohkubo, E., Umino, H., Shioya, M., Tetsui, T., Kimura, R., Naganuma, M., Kato, N., Sato, S., "Investigation of operator-friendly remote control robot system for RAA/RAT using WWW browsing software", Proc. $3^{\text {rd }}$ Int. Conf. on Soft Computing and Intelligent Systems, pp. 1490-1494, 2006.

4. Naganuma, M., Tetsui, T., Ohkubo, E., Kimura, R., Kato, N., Sato, S., "Trial of robot assisted rehabilitation using robotic pet", Tech. Digest. 6th Int. Conf. International Society for Gerontechnology (Pisa, 2008), Paper no. 94-4, 2008.

5. Ohkubo, E., Negishi, T., Oyamada, Y., Kimura, R., Naganuma, M., "Studies on necessary condition of companion robot in the RAA application", Proc. 2003 IEEE Intern. Symp. on Computational Intelli- gence in Robotics and Automation, pp.102-106, 2003

6. Tetsui, T., Ohkubo, E., Murata, H., Kato, N., Wakabayashi, T., Kimura, R., Naganuma, M., "Design and evaluation of simple control console usable by therapist and elderly people in nursing home for robot assisted activity / therapy", Proc. SCIS \& ISIS, Nagoya, pp.720-723, 2008. 